\title{
COLLAGEN HYDROLYSATES PROMOTES LONGITUDINAL BONE GROWTH OF ADOLESCENT RATS
}

\author{
(C) Kim Hye Kyung ${ }^{1}$, Leem Kang-Hyun ${ }^{2}$
}

${ }^{1}$ Department of Food and Biotechnology, Hanseo University, Seosan, Chungnam 356-706, South Korea

${ }^{2}$ College of Oriental Medicine, Semyung University, Jechon, Chungbuk 390-711, South Korea

Collagen hydrolysates $(\mathrm{CH})$ are mixtures of peptides obtained by partial hydrolysis of gelatin that are receiving scientific attention as potential oral supplements for the recovery of osteoarticular tissues. The aim of this study was to provide the $\mathrm{CH}$ which is effective on longitudinal bone growth of growing rats. Porcine skin gelatin was hydrolyzed by manipulating two reaction parameters; enzyme combination and reaction time. An in vitro study was carried out in osteoblast-like MG63 cells and the most effective $\mathrm{CH}$ on bone formation was selected among 36 various $\mathrm{CH}$. The effects of selected $\mathrm{CH}$ (molecular weight of $<3 \mathrm{kDa}$ ) on the rate of longitudinal bone growth of the tibia in the rat were examined. $\mathrm{CH}$ dose-dependently promotes the longitudinal bone growth and height of the growth plate in adolescent male rats whereas gelatin failed to affect longitudinal bone growth. Insulin-like growth factor-1 and bone morphogenetic protein-2 in the $\mathrm{CH}$ treated group were highly expressed in the growth plate. These results suggest that $\mathrm{CH}$ isolated in this study may provide beneficial effects on bone metabolism of growing animals and humans.

\section{EFFECT OF PLANT GROWTH REGULATORS AND BIOTIC ELICITORS ON DICENTRINE PRODUCTION IN HAIRY ROOT CULTURES OF STEPHANIA SUBEROSA FORMAN}

\section{() Kitisripanya Tharita, Yusakul Gorawit, Putalun Waraporn}

${ }^{1}$ Faculty of Pharmaceutical Sciences, Khon Kaen University, Khon Kaen, 40002, Thailand

${ }^{2}$ Research Group for Pharmaceutical Activities of Natural Products using Pharmaceutical

Biotechnology (PANPB), National Research University, Khon Kaen University, Khon Kaen 40002, Thailand

Stephania suberosa Forman is the climber plants with very large tuber in the Menispermaceae and only found in endemic area. Dicentrine, aporphine alkaloids, is the bioactive compound that was found in tuberous root of $S$. suberosa with various pharmacological activities like acetylcholinesterase inhibition, antihypertensive activities, antiarrhythmic effect (1-2). The effect of plant growth regulators (thidiazuron, abscisic acid, forchlorfenuron (CPPU)) and biotic elicitors (methyl jasmonate, yeast extract) at various concentrations on dicentrine production were investigated in hairy root cultures of $S$. suberosa which cultured in a half strength liquid Murashige and Skoog medium. The results of plant growth regulators, thidiazuron at concentration $0.5 \mathrm{mg} / \mathrm{l}$ was obtained the highest dicentrine content $(6.27 \pm 0.40 \mathrm{mg} / \mathrm{g} \mathrm{DW}, \mathrm{p}<0.01)$ on day 20 by time-de- pendent while abscisic acid decreased dicentrine content by dose-dependent. And in this study, all concentrations of CPPU increased dicentrine content on day 5. For biotic elicitors, dicentrine contents in hairy root cultures were decreased by methyl jasmonate and were not significantly increased by yeast extract. In conclusion, long term used thidiazuron, short term used CPPU and lower concentration of abscisic acid may be optimal condition to improve dicentrine production in hairy root cultures of $S$. suberosa.

References: (1) Sriprang S., Khorana N., Ingkaninan K. (2006) Naresuan University Science Journal, 3 (1): 1-11. (2) Lai Y. C., Kuo T. F., Chen C.K., Tsai H.J. and Lee S.S. (2010) Drug Metabolism and Disposition, 38 (10): 17141722. 